\title{
Fixed Versus Adjustable Loop Cortical Suspension Devices in Arthroscopic Anterior Cruciate Ligament Reconstruction
}

\author{
Ramy Said ${ }^{1}$ MD, Mohamed El-Sheikh ${ }^{1}$ MD, Mohamed H. El-Shafie ${ }^{1, *}$ MSc
}

\begin{abstract}
*Corresponding Author: Mohamed El-Shafie

Mohamed El-

Shafieshafei4413@gmail.com Received for publication January2, 2020; accepted January16, 2020, published on line January 25 , 2020.

Copyright 2020 The Authors published by Al-Azhar University, Faculty of Medicine, Cairo, Egypt. All rights reserved. This an openaccess article distributed under the legal terms, where it is permissible to download and share the work provided it is properly cited. The work cannot be changed in anyway or used commercially.

doi: $10.21608 / \mathrm{aimj} .2020 .21868 .104$ 6

${ }^{I}$ Orthopaedic Surgery Department, Faculty of Medicine, Alexandria University Alexandria, Egypt.
\end{abstract}

\begin{abstract}
Background: Anterior cruciate ligament reconstruction has remained the gold (ACL) standard for ACL injuries, especially for young individuals and athletes expose to high level sporting activities aiming to return his or her preinjury level of activity. Cortical suspensory femoral fixation is commonly performed for graft fixation to the femur in anterior cruciate ligament (ACL) reconstruction using hamstring tendons.

Aim of the work: The aim of the work was to compare the clinical results between fixed and adjustable loop cortical suspension devices in arthroscopic ACL reconstruction using the Lysholm Knee Scoring Scale after 12 months postoperatively.

Methods: This study included a total of sixty patients who underwent transportal arthroscopic ACL reconstruction using hamstring tendon autograft from November 2016 to December 2017. For femoral graft fixation, a fixed-length loop device was used in 30 patients (fixed-loop group) and an adjustable-length loop device was used in 30 patients (adjustable-loop group) randomly. For tibial graft fixation, interference screw was used for all patients.

Results: The present study shows that there was no statistically significant difference between the two groups regarding the Lysholm score with highly statistically significant difference between preoperative and postoperative Lysholm score in each group separately.

Conclusions: Both fixed loop and adjustable loop devices in ACL reconstruction provided good clinical outcomes but without significant statistical difference between both groups from the clinical point of view postoperatively using the Lysholm score.
\end{abstract}

Keywords: Anterior cruciate ligament, Suspensory fixation device, adjustable loop, fixed loop.
Disclosure: The authors have no financial interest to declare in relation to the content of this article. The Article Processing Charge was paid for by the authors. Authorship: All authors have a substantial contributions to the article.

\section{INTRODUCTION}

Adequate strong fixation of the ACL graft in both tibial and femoral sides is a must to ensure a good stability of it until the time of biological integration to the bone. ${ }^{1}$ There is no optimal fixation technique of the graft in the terms of the clinical results. ${ }^{2}$ Nowadays, there is a growing trend to use the cortical suspensory fixation devices for the femoral side using transportal techniques for femoral tunnel placement. ${ }^{3-6}$ The fixed loop device has satisfactory biomechanical properties and high failure loads for graft fixation. ${ }^{1,7,8}$ However, it is technically difficult and does not allow the graft to be completely filled in the femoral tunnel socket. Moreover, there is a possibility to make an error in the measurement which may lead to inadequate graft length. Adjustable-Loop fixation devices were designed to solve this problem and to allow the graft to be adapted to the different tunnel lengths. ${ }^{9}$ Although there are no much studies comparing the clinical outcomes of both devices, some biomechanical studies have concluded that there may be a graft slippage in adjustable loop group without retensioning and knot tying. ${ }^{8,10,11,12}$

The hypothesis of this study was that there would be no differences between the two kinds of devices in terms of clinical results.

\section{PATIENTS AND METHODS}

A total of sixty ACL deficient knee patients were included in this study from November 2016 to December 2017. The mean of the age in both groups is 26.5 years and all the patients were males. The inclusion criteria were (1) active patients who wish to continue participating in sport; (2) patients experiencing instability with the daily life activities; (3) patients working in heavy labors who need a stable knee. The exclusion criteria were (1) combined ligamentous injuries; (2) malalignment; (3) previous ACL reconstruction. There was no minimum or maximum time between the injury and the operation. Anterior drawer, Lachman and pivot shift tests were applied to all patients to identify ACL tear which was 
confirmed by MRI scan. The patients were chosen by standard randomisation either to use the fixed-loop or the adjustable-loop. Informed consent was obtained from all the patients in the article.

After clinical and arthroscopic examinations under anesthesia, the hamstring tendons graft were sacrificed and the anterolateral and anteromedial portals were created. To set the ACL graft in the anatomical femoral foot print, an accessory medial portal might be needed to drill the femoral tunnel to the lateral cortex by $4.5 \mathrm{~mm}$ reamer then by 7 to 9 $\mathrm{mm}$ reamer to a depth of $30 \mathrm{~mm}$ according to the graft diameter. Using the ACL tibial C-guide with a $55^{\circ}$, the tibial tunnel was drilled and the ACL graft was passed through the tunnel. The femoral side of the graft was fixed by fixed or adjustable loop (with knot tying) as selected randomly and the tibial side of graft was secured with a bioabsorbable interference screw. (Figures 1, 2)

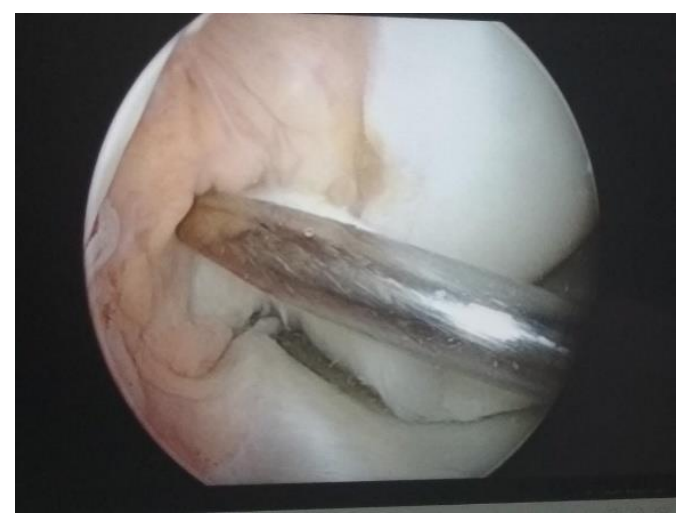

Figure (1): femoral tunnel preparation

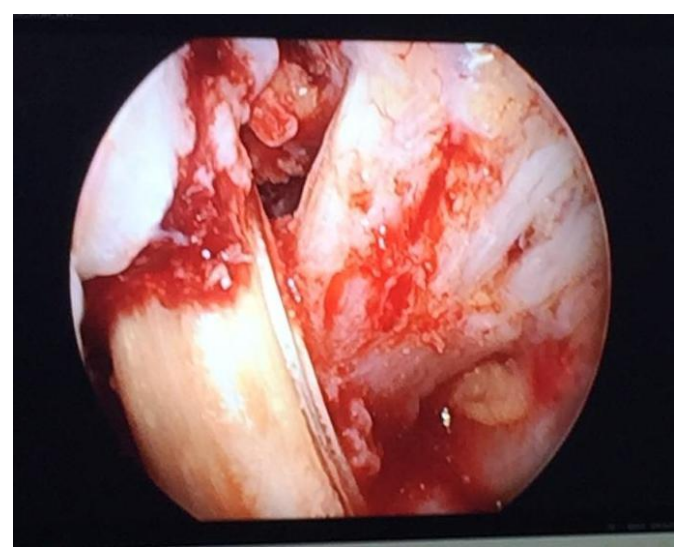

Figure (2): dry arthroscopic view after complete reconstruction limp. Cold therapy, protected full weight bearing as tolerated with crutches, passive ROM $\left(0^{\circ}-90^{\circ}\right)$ and quadriceps muscle exercises were advised from the day of surgery to the end of second postoperative week. From 3rd to 6 th postoperative weeks, nonprotected full weight bearing, gradually full ROM, quadriceps, hamstrings, calf and proprioception exercises were commenced. Full activities were regained usually after 6 months.

At the end of follow-up period which is 12 months postoperatively, all the patients were assessed clinically using the Lysholm Knee Scoring Scale.

\section{RESULTS}

Chi-square test was performed to compare the follow-up data obtained at 12 months after surgery. The Wilcoxon signed-rank test was used to compare pre- and postoperative results of each group. The level of statistical significance was set as $p<0.05$. The mean \pm SD of fixed loop group was $27.23 \pm 3.28$ years old, and adjustable loop groupwas $26.90 \pm 2.47$ years old, and there was no statistical significant difference between groups regarding age. There was no statistical significant difference between two groups regarding associated meniscal injury. There was highly statistical significant difference between preoperative and postoperative regarding to the pain in each group while there was no statistical difference between both groups neither in improvement nor in $\%$ of improvement.

There was highly statistical significant difference between preoperative and postoperative results regarding to swelling in each group while there was no statistical difference between both groups neither in improvement nor in $\%$ of improvement of swelling.

There was no statistical significant difference between lysholm score with age and time laps from injury to surgery in each group.

We had 2 patients in the adjustable loop group with unsatisfactory score because of persistent giving way, locking and difficulty in climbing stair at the end of follow-up and revision was done for both of them.

There was only 1 patient in the fixed loop group with constant pain, swelling and difficult squatting who got unsatisfactory Lysholm score.

Statistically significant improvement in the Lysholm score was observed in each group separately with no statistical significance in comparison between the both groups postoperatively. (Tables 1, 2)
From the time of injury until surgical reconstruction in acute cases, it was important that the knee became quiescent with little or no swelling, had full range of motion and the patient had a normal gait without a 



\begin{tabular}{|l|l|c|c|c|c|}
\hline \multirow{3}{*}{ Lysholm score } & \multicolumn{2}{|c|}{$\begin{array}{c}\text { Adjustable Loop } \\
(\mathbf{n}=\mathbf{3 0})\end{array}$} & \multicolumn{2}{c|}{$\begin{array}{c}\text { Fixed Loop } \\
(\mathbf{n}=\mathbf{3 0})\end{array}$} \\
\cline { 2 - 6 } & & No. & $\mathbf{\%}$ & No. & $\mathbf{\%}$ \\
\hline \multirow{3}{*}{ Pre-operative } & Unsatisfactory & $\mathbf{3 0}$ & $\mathbf{1 0 0 . 0}$ & $\mathbf{3 0}$ & $\mathbf{1 0 0 . 0}$ \\
& Poor (<60) & 21 & 70.0 & 16 & 53.3 \\
& Fair (60-74) & 9 & 30.0 & 14 & 46.7 \\
\hline \multirow{5}{*}{ Post-operative } & Unsatisfactory & $\mathbf{2}$ & $\mathbf{6 . 7}$ & $\mathbf{1}$ & $\mathbf{3 . 3}$ \\
& Poor (<60) & 1 & 3.3 & 0 & 0.0 \\
& Fair (60-74) & 1 & 3.3 & 1 & 3.3 \\
& Satisfactory & $\mathbf{2 8}$ & $\mathbf{9 3 . 3}$ & $\mathbf{2 9}$ & $\mathbf{9 6 . 7}$ \\
& Good (75-90) & 5 & 16.7 & 1 & 3.3 \\
& Excellent (91-100) & 23 & 76.7 & 28 & 93.3 \\
\hline
\end{tabular}

Table 1: Comparison between the two studied groups according to Lysholm score

\begin{tabular}{|c|c|c|c|c|}
\hline Lysholm score & Preoperative & Postoperative & $\mathbf{Z}$ & $\mathbf{P}$ \\
\hline Adjustable loop $(n=30)$ & & & & \\
\hline Min. - Max. & $40.0-74.0$ & $59.0-100.0$ & & \\
\hline Mean \pm SD & $55.20 \pm 9.22$ & $92.97 \pm 9.02$ & $4.784^{*}$ & $<0.001^{*}$ \\
\hline Median & 56.50 & 95.0 & & \\
\hline Improvement & \multicolumn{2}{|c|}{$\uparrow 37.77 \pm 9.59$} & & \\
\hline \% of improvement & \multicolumn{2}{|c|}{$\uparrow 71.79 \pm 26.84$} & & \\
\hline \multicolumn{5}{|l|}{ Fixed $\operatorname{loop}(\mathbf{n}=30)$} \\
\hline Min. - Max. & $43.0-71.0$ & $66.0-100.0$ & & \\
\hline Mean \pm SD & $57.47 \pm 7.36$ & $95.03 \pm 6.39$ & $4.787^{*}$ & $<0.001^{*}$ \\
\hline Median & 59.0 & 95.0 & & \\
\hline Improvement & \multicolumn{2}{|c|}{$\uparrow 37.57 \pm 7.09$} & & \\
\hline \% of improvement & \multicolumn{2}{|c|}{$\uparrow 67.34 \pm 19.27$} & & \\
\hline $\mathbf{p}_{1}$ & \multicolumn{2}{|c|}{0.959} & & \\
\hline $\mathbf{p}_{2}$ & \multicolumn{2}{|c|}{0.574} & & \\
\hline
\end{tabular}

Table 2: The statistical difference between the two studied groups according to Lysholm score $\mathrm{Z}$ : Wilcoxon signed ranks test $\mathrm{p}$ : $\mathrm{p}$ value for comparing between preoperative and postoperative p1: $\mathrm{p}$ value for Mann Whitney test for comparing between group 1 and group 2 in improvement $\mathrm{p} 2$ : $\mathrm{p}$ value for Mann Whitney for comparing between group 1 and group 2 in $\%$ of improvement *: Statistically significant at $\mathrm{p} \leq 0.05$

\section{DISCUSSION}

ACL reconstruction is very important to maintain a stable knee and prevent the arthritic changes that might occur in ACL deficient knees. Hamstring tendon graft is more popular to reconstruct the ACL due to its positive clinical outcomes as reported by many authors. ${ }^{12-14}$

Suspensory fixation devices have a button to rest on the lateral femoral cortex and a loop that holds the graft inside the tunnel until bone integration occurs. Many surgeons prefer to use these devices because of their high biomechanical properties and less chances of the intraarticular damage. ${ }^{15,16}$ Fixed loop device does not permit the graft to completely fill the tunnel with the possibility of synovial fluid leakage which interfere with the graft bone integration. Over the fixed loop, adjustable loop has the advantages of tensioning the graft after fixation, flexibility of the length of the femoral tunnel and complete filling of the tunnel by the graft. ${ }^{17}$

Eguchi et al. and Barrow et al. concluded that the adjustable loop is not mechanically as strong as the fixed loop with high liability of loop lengthening and slippage. ${ }^{12,18}$ Our in vivo clinical results do not match with their in vitro results. Our study demonstrates that there is a significant improvement in each group in terms of Lysholm score with no statistically significant difference between both groups at 12 months postoperatively indicating that the adjustable-length loop does not loosen after ACL reconstruction. These clinical results are supported by several studies that showed no clinical differences among the suspensory femoral fixation 
devices. Boyle et al. reported that there is no difference between fixed and adjustable loop regarding the postoperative clinical results, knee laxity or graft failure rates at a period of 2-year follow-up. ${ }^{19}$ Choi et al. demonstrated that there is better stability with pivot-shift test(rotatory instability) in fixed loop group and with Lachman test(anteroposterior instability) in adjustable loop group. ${ }^{10}$ Ahn et al. concluded that there is no statistical difference between fixed and adjustable loop in clinical outcomes and the degree of graft displacement by postoperative MRI evaluation. ${ }^{20} \mathrm{He}$ also said that there was no statistical significant difference with associated meniscal injuries regarding the postoperative clinical results between the both groups. ${ }^{20}$ The present study also shows that there was no statistically significant difference between the two groups regarding associated meniscal injury.

This study has few limitations as the clinical outcome was assessed subjectively without the use of arthrometer. Also, no MRI was available postoperatively at the end of the follow-up period.

\section{CONCLUSION}

Cortical suspensory fixation devices are very effective methods for arthroscopic ACL reconstruction. Although they have different biomechanical aspects, the clinical outcomes are the same. Proper technique with appropriate tunnel positioning are the main factors of a satisfied patient functionally following ACL reconstruction.

\section{REFERENCES}

1. Kousa P, Jarvinen TL, Vihavainen M, et al. The Fixation Strength of Six Hamstring Tendon Graft Fixation Devices in Aanterior Cruciate Ligament Reconstruction. Part II: tibial site. Am J Sports Med. 2003;31(2):182-8.

2. Brown CH Jr, Wilson DR, Hecker AT, et al. bone Motion and Tensile Properties of Hamstring and Patellar Tendon Anterior Cruciate Ligament Femoral Graft Fixation under Cyclic Loading. Arthroscopy. 2004;20(9):922-35.

3. Lee S, Ko S and Ahn H. Comparison of tibial tunnel techniques in posterior cruciate ligament reconstruction: C-arm versus anatomic fovea landmark. Arthroscopy. 2016;32(3):487-92.

4. Lee S, Lee K, Oh S, et al. Comparison of femoral tunnel widening between outside-in and trans-tibial double bundle ACL reconstruction. Knee Surg Sports Traumatol Arthrosc. 2014;22(9):2033-9.

5. Ahn H, Lee S and Lee H. Creation of an anatomic femoral tunnel with minimal damage to the remnant bundle in remnant-preserving anterior cruciate ligament reconstructionusing an outside-in technique. Arthrosc Tech. 2014;3(1):e175-9.

6. Lee S, Lee K and Chun I. Flipping method of a Retro Button during ACL reconstruction with outside-in femoral drilling using a Flip Cutter. Orthopedics. 2012;35(10):861-4.

7. Kamelger S, Onder U, Schmoelz W, et al. Suspensory fixation of grafts in anterior cruciate ligament reconstruction: a biomechanical comparison of 3 implants. Arthroscopy. 2009;25(7):767-76.

8. Petre M, Smith D and Jansson S. Femoral cortical suspension devices for soft tissue anterior cruciate ligament reconstruction: a comparative biomechanical study. Am J Sports Med. 2013;41(2):416-22.

9. Conner S, Perez A, Morris P, et al. Three femoral fixation devices for anterior cruciate ligament reconstruction: comparison of fixation on the lateral cortex versus the anterior cortex. Arthroscopy. 2010;26(6):796-807.

10. Choi H, Yang $S$ and Victoroff N. Clinical and radiological outcomes after hamstring anterior cruciate ligament reconstructions: comparison between fixedloop and adjustable- loop cortical suspension devices. Am J Sports Med. 2017;45(4):826-31.

11. De Berardino M, Smith A and Cook L. Femoral suspension devices for anterior cruciate ligament reconstruction: letter to the editor. Am J Sports Med. 2014;42(2):NP15-6.

12. Eguchi A, Ochi M, Adachi N, et al. Mechanical properties of suspensory fixation devices for anterior cruciate ligament reconstruction: comparison of the fixed-length loop device versus the adjustable-length loop device. Knee. 2014;21(3):743-8.

13.Ferretti A, Conteduca F, Morelli F, et al. Biomechanics of anterior cruciate ligament reconstruction using twisted doubled hamstring tendons. Int Orthop. 2003; 27:22-25. 24.

14. Aglietti P, Buzzi R, Zaccherotti G, et al. Patellar tendon versus doubled semitendinosus and racilis tendons for anterior cruciate ligament reconstruction. Am J Sports Med. 1994;22:211-17.

15. Chang B, Choi Y, Koh J,et al. Comparisons of femoral tunnel position and length in anterior cruciate ligament reconstruction: modified transtibial versus anteromedial portal techniques. Arthroscopy. 2011;27(10):1389.

16. Wang H, Kim G, Lee K, et al. Comparison of femoral graft bending angle and tunnel length between transtibial technique and transportal technique in anterior cruciate ligament reconstruction. Knee Surg Sports Traumatol Arthrosc. 2012;20(August (8)):1584-93.

17. Lubowitz H, Ahmad S and Anderson K. All-inside anterior cruciate ligament graft-link technique: secondgeneration, no incision anterior cruciate ligament reconstruction. Arthroscopy. 2011;27:717-27.

18. Barrow AE, Pilia M, Guda T and Kadrmas WR, Burns TC. Femoral suspension devices for anterior cruciate ligament reconstruction: do adjustable loops lengthen? Am J Sports

Med. 2014;42(2):343-9.

19. Boyle J, Vovos J, Walker G, et al. Does adjustable-loop femoral cortical suspension loosen after anterior cruciate ligament reconstruction? A retrospective comparative study. Knee. 2015;22(4):304-8.

20. Ahn H, Ko S, Lee S, et al. Magnetic Resonance Imaging and Clinical Results of Outside-in Anterior Cruciate Ligament Reconstruction: A Comparison of Fixed and Adjustable-Length Loop Cortical Fixation. Clin Orthop Surg 2018; 157-66. 